These effects can be produced in seven days or less, too soon for qualitative malnutrition to affect the animal's physical well-being ; indeed it remains in perfect health, being supplied with everything necessary for healthy functioning, except, during a few days only, ascorbic acid. It follows that reduction or disappearance of "complement" from the circulating blood must be one of the earliest signs (in guinea pigs, and perhaps in man) of a state of affairs which, if prolonged for twenty to forty days (in the cavy) or four to eight months (in man) will result in scurvy.

Pathological Laboratory, Frank Marsh.

$$
\begin{gathered}
\text { c/o Anglo-Iranian Oil Co., Ltd., } \\
\text { Masjid-i-Sulaiman, } \\
\text { Via Ahwaz, South Iran. }
\end{gathered}
$$$$
\text { Feb. } 8 .
$$

${ }^{1}$ Cadham, F., "Septicaemia : a Method of Treatment", Amer. J. Med. Sci., 188, 542 (1934).

${ }^{2}$ Whitby, L. E. H., and Britton, C. J. C., "Disorders of the Blood", 45 (J. and A. Churchill, London, 1935).

${ }^{3}$ Gordon, J., and Wormall, A., "The Action of Ultra-Violet Rays on Complement", Biochem. J., 22 (4), 909 (1928).

"Gordon, J., and Thompson, F. C., "The Relationship between the Complement and Opsonin of Normal Serum", Brit. J. Exp. Path., $16(1), 101$ (1935).

\section{A Satisfactory Substitute for the Osmium Tetroxide Golgi Apparatus Methods}

Osmium tetroxide at 18s, a gram is the most expensive substance used in biological research. It is principally used in the Kolatchew and Weigl methods for the Golgi apparatus, and in the F. W. A. and Champy methods for mitochondria. The nearest cheap substitutes for Weigl and Kolatchew methods are the formalin-silver methods of Cajal and Da Fano. The trouble with these latter methods is that there is usually distortion and often incrustation of the Golgi bodies, banana-shaped elements, for example, often becoming beads, or spheres, and different from what can be seen intra vitam. The general fixation is often very poor.

I have been astonished at the beauty of silver nitrate formalin preparations made according to F. Aoyama's modification of Cajal's method ${ }^{\mathbf{1}}$. This method is as follows : Fix small pieces of tissue in cadmium chloride 1 part, formol neutral 15 parts, distilled water 85 parts, for three to four hours. Rinse quickly in two changes of distilled water, and transfer to 1.5 per cent solution of silver nitrate for 10 to 15 hours at $22^{\circ} \mathrm{C}$. Rinse quickly in two changes of distilled water, preferably in a darkened room, and transfer for 5 to 10 hours to the reducing solution (hydroquinone 1 part, neutral formol 15 parts, distilled water 85 parts, 0.1 to 0.15 parts of sodium sulphite, sufficient to produce yellowish tinge). Wash thoroughly in tap water ( $\frac{1}{2}$ to $\frac{3}{4}$ of an hour), upgrade, imbed and section. Counterstain in carmine or hæmatoxylin and eosin. Cold-blooded animals may need longer impregnation and fixation.

This method has been tried in this laboratory on the following material : rat pancreas and dorsal root ganglion, Helix ovotestis and cerebral ganglion, midgut of mealworm. In every case the preparations were superior to those got by Da Fano's method, and very nearly approached the best Weigl preparations for morphology of Golgi bodies. The noncellular elements of tissues were bright yellow, and quite different from the Cajal or Da Fano effect. The rat and snail preparations were perfect enough for the most precise research on cell inclusions. In only a few cases did mitochondria impregnate, and the method is probably more specific than Weigl, and much more specific than Da Fano.

The preparations were made by Mr. R. Brown and Miss M. Daniels. The formalin used was unneutralised, but from a fresh supply; the silvering was done in a Hearson incubator at the proper temperature.

\section{Trinity College,}

J. Brontë Gatensy.

$$
\text { Dublin. }
$$

Feb. 24.

'Z. wiss. Mikr., (1930).

\section{Induction of Mammary Ducts}

THe mammary gland of the pig has normally two primary ducts which open separately on the surface of the nipple. Embryologically, the two ducts proliferate from the base of a cone-shaped depression of the stratum germinativum known as the mammary pocket or mammary bud. A similar depression or epithelial ingrowth, variable in depth, surrounds the nipple at its juncture with the body wall. Among several foetal glands sectioned, a few cases of aberrant ducts, similar histologically to the primary ducts, were noticed to have sprouted from the same relative position to the apex of the epithelial ingrowth at the base of the nipple as the primary ducts do to the apex of the mammary pocket (Fig. 1).

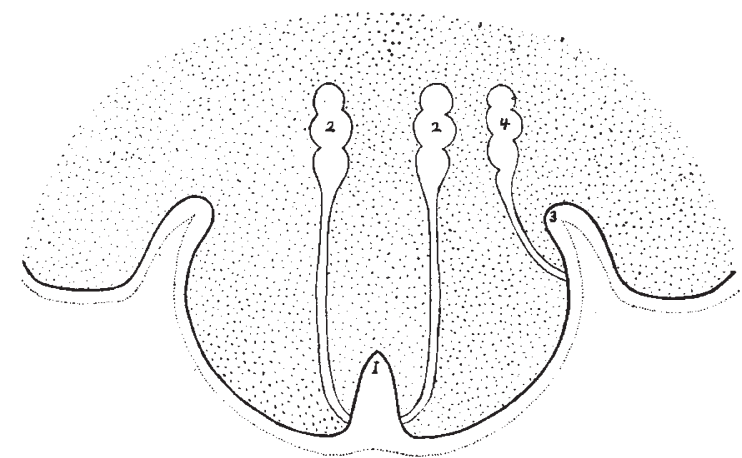

FIG. 1. Diagrammatic section through the nipple of a foetal pig. (1) Mammary pocket; (2) primary ducts ; (3) epithelial ingrowth; (4) aberrant duct.

These aberrant ducts would appear to furnish evidence that an inducing stimulus is responsible for the development of the primary ducts, similar in principle to Spemann's demonstration ${ }^{1}$ of the underlying mesoderm providing an inducing stimulus for neural plate development in Urodela. Recently, Waddington ${ }^{2}$ suggested that some, if not all, inductions are due to the diffusion of active chemical substances into the reacting tissues. It would appear reasonable therefore to infer that an active substance is present in the nipple which stimulates duct develop. ment in specific locations relative to apices of epithelial ingrowths. Furthermore, that the stimulus is specific for particular groups of animals. Thus in the horse, in common with the pig, there are normally two primary ducts which develop respectively from the anterior and posterior walls of the mammary pocket; ruminants have only one duct, which proliferates from the apex of the mammary pocket, and in man there are several primary ducts. 